\title{
Pacific
}

Journal of

Mathematics

\section{EXTREMAL FUNCTIONS AND THE CHANG-MARSHALL INEQUALITY}

\author{
Valentin V. AndReEV AND Alec Lane Matheson
}




\title{
EXTREMAL FUNCTIONS \\ AND THE CHANG-MARSHALL INEQUALITY
}

\author{
Valentin V. Andreev And Alec Matheson
}

Answering a question of J. Moser, S.-Y. A. Chang and D. E. Marshall proved the existence of a constant $C$ such that $\frac{1}{2 \pi} \int_{0}^{2 \pi} e^{\left|f\left(e^{i \theta}\right)\right|^{2}} d \theta$ $\leq C$ for all functions $f$ analytic in the unit disk with $f(0)=0$ and Dirichlet integral not exceeding one. We show that there are extremal functions for the functionals $\Lambda_{\alpha}(f)=\frac{1}{2 \pi} \int_{0}^{2 \pi} e^{\alpha\left|f\left(e^{i \theta}\right)\right|^{2}} d \theta$ when $0 \leq \alpha<1$. We establish a variational condition satisfied by extremal functions. We show that the identity function $f(z)=z$ is a local maximum in a certain sense for the functionals $\Lambda_{\alpha}$ and conjecture that it is a global maximum.

1. Introduction. The Dirichlet space $\mathfrak{D}$ consists of those functions $f$ analytic on the unit disk $\Delta$ which have finite Dirichlet integral

$$
\|f\|_{\mathfrak{D}}^{2}=\frac{1}{\pi} \iint_{\Delta}\left|f^{\prime}(z)\right|^{2} d x d y .
$$

We will always assume that $f(0)=0$. It is well-known and easy to establish that $\mathfrak{D}$ is a Hilbert space under the inner product

$$
\langle f, g\rangle=\frac{1}{\pi} \iint_{\Delta} f^{\prime}(z) \overline{g^{\prime}(z)} d x d y,
$$

and that, if $f(z)=\sum_{n=1}^{\infty} a_{n} z^{n}$ and $g(z)=\sum_{n=1}^{\infty} b_{n} z^{n}$, then

$$
\langle f, g\rangle=\sum_{n=1}^{\infty} n a_{n} \overline{b_{n}} \text {. }
$$

In particular,

$$
\|f\|_{\mathfrak{D}}^{2}=\sum_{n=1}^{\infty} n\left|a_{n}\right|^{2} .
$$

For $\alpha \geq 0$ and $f \in \mathfrak{D}$, we define

$$
\Lambda_{\alpha}(f)=\frac{1}{2 \pi} \int_{0}^{2 \pi} e^{\alpha\left|f\left(e^{i \theta}\right)\right|^{2}} d \theta
$$

This is known to be finite for all $\alpha \geq 0$ and all $f \in \mathfrak{D}$, and it can be shown that the quantity

$$
I_{\alpha}=\sup \left\{\Lambda_{\alpha}(f) \mid\|f\|_{\mathfrak{D}} \leq 1\right\}
$$


is finite for $0 \leq \alpha \leq 1$ and infinite for $\alpha>1$. A proof of this for $0 \leq \alpha<1$ will be indicated below. For $\alpha>1$, this follows from an estimate of A. Beurling [1]. Answering a question of J. Moser [9], the finiteness of this quantity for the critical value $\alpha=1$ was first established by S.-Y. A. Chang and D. E. Marshall [5]. A different proof of this fact was subsequently given by Marshall [8], and this has been generalized by $\mathbf{M}$. Essén [7].

The proof of Chang and Marshall involves a delicate argument based on an estimate of Beurling [1]. Since we need Beurling's estimate in section 2 , we describe it here. Let $f \in \mathfrak{D}$ satisfy $\|f\|_{\mathfrak{D}} \leq 1$, and for $t \geq 0$, let

$$
E_{t}=\left\{\theta|| f\left(e^{i \theta}\right) \mid>t\right\} .
$$

If $\left|E_{t}\right|$ denotes the normalized Lebesgue measure of $E_{t}$, then Beurling's estimate is

$$
\left|E_{t}\right| \leq e^{-t^{2}+1}
$$

It follows immediately that

$$
\begin{aligned}
\Lambda_{\alpha}(f) & =1+2 \alpha \int_{0}^{\infty} t e^{\alpha t^{2}}\left|E_{t}\right| d t \\
& \leq 1+2 \alpha e \int_{0}^{\infty} t e^{-(1-\alpha) t^{2}} d t=1+e \frac{\alpha}{1-\alpha},
\end{aligned}
$$

if $0 \leq \alpha<1$. We note that this proves that $I_{\alpha}$ is finite if $0 \leq \alpha<1$, but, in light of the result of Chang and Marshall, the bound is far from precise.

We now show that $\Lambda_{\alpha}(f)$ is finite for any $f \in \mathfrak{D}$ and any $\alpha>0$. By scaling, it is clear that $\Lambda_{\alpha}(f)<\infty$ if $\alpha\|f\|_{\mathfrak{D}}^{2}<1$. If $f \in \mathfrak{D}$ and $\epsilon>0$, there is a polynomial $p$ such that $\|f-p\|_{\mathfrak{D}}^{2}<$ $\epsilon$. But $|f|^{2} \leq 2|f-p|^{2}+2|p|^{2} \leq 2|f-p|^{2}+2\|p\|_{\infty}^{2}$, where $\|p\|_{\infty}=$ $\sup \{|p(z)| \mid z \in \Delta\}$. Then

$$
\Lambda_{\alpha}(f) \leq e^{2 \alpha\|p\|_{\infty}} \frac{1}{2 \pi} \int_{0}^{2 \pi} e^{2 \alpha|f-p|^{2}} d \theta,
$$

and, by taking $\epsilon$ small enough, it follows that $\Lambda_{\alpha}(f)$ is finite. Chang and Marshall attribute this observation to J. Garnett. We also note that, since $x^{n} e^{x^{2}} \leq C_{n} e^{2 x^{2}}$ for any positive integer and some constant $C_{n}$, the quantity

$$
\frac{1}{2 \pi} \int_{0}^{2 \pi} p\left(\left|f\left(e^{i \theta}\right)\right|\right) e^{\alpha\left|f\left(e^{i \theta}\right)\right|^{2}} d \theta
$$

is finite for any $\alpha>0$, any $f \in \mathfrak{D}$, and any polynomial $p$. 
It is natural to ask if there exist extremal functions for the functionals $\Lambda_{\alpha}$ in case $0<\alpha \leq 1$. In the subcritical case $0<\alpha<1$, this will be established by an easy continuity argument in $\S 2$. The critical case $\alpha=1$ appears to be much more difficult, and the remainder of this paper is devoted to studying this problem. In $\S 3$ we establish a variational criterion which must be satisfied by extremal functions which are sufficiently smooth. It will be seen that the identity function $f(z)=z$ satisfies this criterion. In $\S 4$ we show that in a certain sense the identity function is a local maximum for the functionals $\Lambda_{\alpha}$. In $\S 5$ we discuss various other aspects of this problem.

Finally, we make two observations concerning the nature of extremal functions. The first of these is attributed by Chang and Marshall to J. Clunie.

Let $f(z)=\sum_{n=1}^{\infty} a_{n} z^{n}$ have finite Dirichlet integral, and let $f^{*}(z)=$ $\sum_{n=1}^{\infty}\left|a_{n}\right| z^{n}$. Then clearly $\|f\|_{\mathfrak{D}}=\left\|f^{*}\right\|_{\mathfrak{D}}$, while

$$
\begin{aligned}
\frac{1}{2 \pi} \int_{0}^{2 \pi}\left|f\left(e^{i \theta}\right)\right|^{2 n} d \theta & =\sum_{k=1}^{\infty}\left|\sum_{j_{1}+\cdots+j_{n}=k} a_{j_{1}} \ldots a_{j_{n}}\right|^{2} \\
& \leq \sum_{k=1}^{\infty}\left(\sum_{j_{1}+\cdots+j_{n}=k}\left|a_{j_{1}}\right| \ldots\left|a_{j_{n}}\right|\right)^{2} \\
& =\frac{1}{2 \pi} \int_{0}^{2 \pi}\left|f^{*}\left(e^{i \theta}\right)\right|^{2 n} d \theta
\end{aligned}
$$

for $n=1,2, \ldots$. It follows from the power series representation $\Lambda_{\alpha}(f)=\sum_{n=0}^{\infty} \alpha^{n} \frac{\|f\|_{2 n}^{2 n}}{n !}$ that $\Lambda_{\alpha}(f) \leq \Lambda_{\alpha}\left(f^{*}\right)$. Because of this, it is enough to consider functions with nonnegative coefficients.

The second observation uses a theorem of L. Carleson [2] to show that extremal functions must be of a certain form. Although we do not use this observation in the sequel, we think it is of sufficient interest to include here. It is clear that if $f$ is extremal for the functional $\Lambda_{\alpha}$, then $\|f\|_{\mathfrak{D}}=1$. Since $f$ belongs to the Hardy space $H^{2}, f$ admits a Riesz factorization

$$
f(z)=z^{m} B(z) S(z) F(z),
$$

where $m$ is a positive integer, $B(z)$ is a Blaschke product, $S(z)$ is a singular inner function, and $F(z)$ is an outer function [6, Thm. 2.8]. Let $g(z)=z F(z)$. Then Carleson's theorem guarantees that $\|g\|_{\mathfrak{D}} \leq\|f\|_{\mathfrak{D}}$, with equality if and only if $f=g$. On the other hand, $\left|f\left(e^{i \theta}\right)\right|=\left|g\left(e^{i \theta}\right)\right|$ almost everywhere, so $\Lambda_{\alpha}(f)=\Lambda_{\alpha}(g)$. Thus an 
extremal function must have the form $z F(z)$, where $F(z)$ is an outer function.

2. Weak continuity of functionals. Let $B$ denote the closed unit ball of $\mathfrak{D}$. Since $B$ is weakly compact, in order to prove the existence of an extremal function for $\Lambda_{\alpha}$, it is sufficient to show that $\Lambda_{\alpha}$ is weakly continuous on $B$. Since

$$
\Lambda_{\alpha}(f)=\sum_{n=0}^{\infty} \alpha^{n} \frac{\|f\|_{2 n}^{2 n}}{n !}
$$

for each $f \in B$. We can do this for $0<\alpha<1$ by showing that the $L^{p}$ norms $f \rightarrow\|f\|_{p}$ are weakly continuous on $B$, and by establishing an estimate $\|f\|_{2 n}^{2 n}=O(n !)$ in order to get uniform convergence of the series above. This is done with the following three lemmas. In what follows, we use standard notation and ideas from the theory of Hardy spaces as found, for example, in [6]. In particular, we note that the norm of a function $f(z)$ in the Hardy space $H^{p}$ coincides with the norm of its boundary function $f\left(e^{i \theta}\right)$ in $L^{p}$.

LEMMA 1. If $f \in \mathfrak{D}$, then $f \in H^{p}$, and

$$
\|f\|_{p}^{p} \leq e \frac{p}{2} \Gamma\left(\frac{p}{2}\right)\|f\|_{\mathfrak{D}}
$$

Proof. Let $f \in \mathfrak{D}$ satisfy $\|f\|_{\mathfrak{D}}=1$. Let $E_{t}=\left\{\theta|| f\left(e^{i \theta}\right) \mid>t\right\}$. Then

$$
\begin{aligned}
\|f\|_{p}^{p} & =p \int_{0}^{\infty} t^{p-1}\left|E_{t}\right| d t \leq e p \int_{0}^{\infty} t^{p-1} e^{-t^{2}} d t \\
& =e \frac{p}{2} \int_{0}^{\infty} u^{\frac{p}{2}-1} e^{-u} d u=e \frac{p}{2} \Gamma\left(\frac{p}{2}\right),
\end{aligned}
$$

where we have used Beurling's estimate $\left|E_{t}\right| \leq e^{-t^{2}+1}$. The lemma follows.

LEMMA 2. If $\left(f_{n}\right)_{n=1}^{\infty}$ is a weakly null sequence in $\mathfrak{D}$, then $\left\|f_{n}\right\|_{2} \rightarrow$ 0 .

Proof. Since weakly null sequences are bounded, we may assume that $f_{n} \in B$ for each $n$. Writing $f_{n}(z)=\sum_{k=1}^{\infty} a_{n, k} z^{k}$, we have

$$
\|f\|_{\mathfrak{D}}^{2}=\sum_{k=1}^{\infty} k\left|a_{n, k}\right|^{2},
$$


and

$$
\|f\|_{2}^{2}=\sum_{k=1}^{\infty}\left|a_{n, k}\right|^{2} .
$$

For any positive integer $K$, the Cauchy-Schwarz inequality yields

$$
\begin{aligned}
\sum_{k=K}^{\infty}\left|a_{n, k}\right|^{2} & \leq\left(\sum_{k=K}^{\infty} \frac{1}{k}\left|a_{n, k}\right|^{2}\right)^{1 / 2}\left(\sum_{k=K}^{\infty} k\left|a_{n, k}\right|^{2}\right)^{1 / 2} \\
& \leq \frac{1}{\sqrt{K}}\left(\sum_{k=K}^{\infty}\left|a_{n, k}\right|^{2}\right)^{1 / 2},
\end{aligned}
$$

so that

$$
\sum_{k=K}^{\infty}\left|a_{n, k}\right|^{2} \leq \frac{1}{K}
$$

for $n=1,2, \ldots$. On the other hand, because $\left(f_{n}\right)_{n=1}^{\infty}$ is weakly null, for each fixed $k, a_{n, k} \rightarrow 0$ as $n \rightarrow \infty$. Hence, given $\epsilon>0$, we can choose $K$ so that $\frac{1}{K}<\frac{\epsilon}{2}$, and then choose $N$ so that if $n \geq N$ and $k<K$, then $\left|a_{n, k}\right|<\frac{\epsilon}{2 K}$. Hence, if $n \geq N,\|f\|_{2}^{2}<\epsilon$ and the lemma is proved.

Lemma 3. For each $p, 0<p<\infty$, the function $f \rightarrow\|f\|_{p}$ is weakly continuous on $B$.

Proof. It is enough to show that if $f_{n} \rightarrow f$ weakly in $B$, then $\left\|f_{n}-f\right\|_{p} \rightarrow 0$. Let $g_{n}=\frac{1}{2}\left(f_{n}-f\right)$. Then $g_{n} \in B$, and $\left(g_{n}\right)_{n=1}^{\infty}$ is weakly null, so $\left\|g_{n}\right\|_{2} \rightarrow 0$ by Lemma 2 . If $0<p<2$, then $\left\|g_{n}\right\|_{p} \leq\left\|g_{n}\right\|_{2}$ by Hölder's inequality, and the lemma is immediate. For $p>2$, we use Hölder's inequality in the form

$$
\|f\|_{p} \leq\|f\|_{2}^{\frac{1}{p}}\|f\|_{2 p-2}^{1-\frac{1}{p}}
$$

together with Lemma 1 to complete the proof.

We have now established the following theorem

THEOREM 1. For each $\alpha, 0<\alpha<1$, the nonlinear functional

$$
\Lambda_{\alpha}(f)=\frac{1}{2 \pi} \int_{0}^{2 \pi} e^{\alpha\left|f\left(e^{i \theta}\right)\right|^{2}} d \theta
$$

is weakly continuous on $B$. Consequently $\Lambda_{\alpha}$ attains its maximum on $B$.

3. A variational condition. If the function $f \in \mathfrak{D},\|f\|_{\mathfrak{D}}=1$, is extremal for some $\Lambda_{\alpha}, 0<\alpha \leq 1$, and if $f$ has a certain degree of 
smoothness up to the boundary of $\Delta$, then it is possible to establish a condition satisfied by $f$ by means of a variational argument. In order for the integrals below to be defined we will assume that $f^{\prime}$ belong to the Hardy space $H^{1}$. In this case $f$ will be continuous on the closed disk, and it will be sufficient to assume that the test functions $\phi$ also have derivatives in $H^{1}$.

Proposition 1. Suppose that $f \in \mathfrak{D},\|f\|_{\mathfrak{D}}=1, f^{\prime} \in H^{1}$, and $f$ is extremal for the functional $\Lambda_{\alpha}$, for some $\alpha, 0<\alpha \leq 1$. Let $\phi$ be a function with $\phi^{\prime} \in H^{1}$. Then

$$
\begin{aligned}
& \frac{1}{2 \pi} \int_{0}^{2 \pi}\left|f\left(e^{i \theta}\right)\right|^{2} e^{\alpha\left|f\left(e^{i \theta}\right)\right|^{2}} 2 \Re \phi\left(e^{i \theta}\right) d \theta \\
& \quad=\frac{S_{\alpha}(f)}{2 \pi} \int_{0}^{2 \pi}\left\{2 \Re \phi\left(e^{i \theta}\right) f^{\prime}\left(e^{i \theta}\right) \overline{f\left(e^{i \theta}\right)}+\phi^{\prime}\left(e^{i \theta}\right)\left|f\left(e^{i \theta}\right)\right|^{2}\right\} e^{i \theta} d \theta
\end{aligned}
$$

where

$$
S_{\alpha}(f)=\frac{1}{2 \pi} \int_{0}^{2 \pi}\left|f\left(e^{i \theta}\right)\right|^{2} e^{\alpha\left|f\left(e^{i \theta}\right)\right|^{2}} d \theta
$$

Proof. Write $\phi=u+i v$, and let $f_{t}=f e^{t \phi}$ for real $t$. Let

$$
h(t)=\frac{1}{2 \pi} \int_{0}^{2 \pi} \exp \left\{\alpha \frac{\left|f_{t}\left(e^{i \theta}\right)\right|^{2}}{\left\|f_{t}\right\|_{\mathfrak{D}}^{2}}\right\} d \theta .
$$

Then, differentiating under the integral sign, we obtain

$$
h^{\prime}(t)=\frac{1}{2 \pi} \int_{0}^{2 \pi} \exp \left\{\alpha \frac{\left|f_{t}\left(e^{i \theta}\right)\right|^{2}}{\left\|f_{t}\right\|_{\mathfrak{D}}^{2}}\right\} \frac{\partial}{\partial t} \frac{\alpha\left|f_{t}\left(e^{i \theta}\right)\right|^{2}}{\left\|f_{t}\right\|_{\mathfrak{D}}^{2}} d \theta
$$

Because $f$ is extremal, $h^{\prime}(0)=0$, and it will be necessary to evaluate the integrand when $t=0$. Since $f_{0}=f$, and $\|f\|_{\mathfrak{D}}=1$, the first factor becomes $\exp \left\{\alpha\left|f\left(e^{i \theta}\right)\right|^{2}\right\}$ when $t=0$. Since $\left|f_{t}\right|^{2}=|f|^{2} e^{2 t u}$, we have

$$
\frac{\partial}{\partial t} \frac{\left|f_{t}\right|^{2}}{\left\|f_{t}\right\|_{\mathfrak{D}}^{2}}=\frac{2 u|f|^{2} e^{2 u t}}{\left\|f_{t}\right\|_{\mathfrak{D}}^{2}}-\frac{\left|f_{t}\right|^{2}}{\left\|f_{t}\right\|_{\mathfrak{D}}^{4}} \frac{\partial}{\partial t}\left\|f_{t}\right\|_{\mathfrak{D}}^{2}
$$

and the first term becomes $2 u|f|^{2}$ when $t=0$. By Green's Theorem,

$$
\left\|f_{t}\right\|_{\mathfrak{D}}^{2}=\frac{1}{2 \pi} \int_{0}^{2 \pi} f_{t}^{\prime}\left(e^{i \theta}\right) \overline{f_{t}\left(e^{i \theta}\right)} e^{i \theta} d \theta
$$

and so

$$
\frac{\partial}{\partial t}\left\|f_{t}\right\|_{\mathfrak{D}}^{2}=\frac{1}{2 \pi} \int_{0}^{2 \pi}\left\{\frac{\partial f_{t}^{\prime}}{\partial t} \overline{f_{t}}+f_{t}^{\prime} \frac{\partial \overline{f_{t}}}{\partial t}\right\} e^{i \theta} d \theta
$$


But

$$
f_{t}^{\prime}=f^{\prime} e^{t \phi}+t \phi^{\prime} f e^{t \phi}
$$

so

$$
\frac{\partial f_{t}^{\prime}}{\partial t}=\phi f^{\prime} e^{t \phi}+\phi^{\prime} f e^{t \phi}+t \phi \phi^{\prime} e^{t \phi}
$$

Hence,

$$
\left.\frac{\partial f_{t}^{\prime}}{\partial t}\right|_{t=0}=\phi f^{\prime}+\phi^{\prime} f
$$

and so

$$
\left.\frac{\partial f_{t}^{\prime}}{\partial t} \overline{f_{t}}\right|_{t=0}=\phi \bar{f} f^{\prime}+\phi^{\prime} \overline{f f}
$$

Also,

$$
\frac{\partial f_{t}}{\partial t}=\phi f e^{t \phi}
$$

so that

$$
\left.f_{t}^{\prime} \frac{\partial \overline{f_{t}}}{\partial t}\right|_{t=0}=f^{\prime} \overline{\phi f}
$$

Consequently, with $z=e^{i \theta}$,

$$
\begin{aligned}
\left.\frac{\partial}{\partial t}\left\|f_{t}\right\|_{\mathfrak{D}}^{2}\right|_{t=0} & =\frac{1}{2 \pi} \int_{0}^{2 \pi}\left(\phi \bar{f} f^{\prime}+\phi^{\prime} \overline{f f}+\overline{\phi f} f^{\prime}\right) z d \theta \\
& =\frac{1}{2 \pi} \int_{0}^{2 \pi}\left(2 u \bar{f} f^{\prime}+\phi^{\prime} \overline{f f}\right) z d \theta
\end{aligned}
$$

Hence,

$$
\left.\frac{\partial}{\partial t} \frac{\left|f_{t}\right|^{2}}{\left\|f_{t}\right\|_{\mathfrak{D}}^{2}}\right|_{t=0}=2 u|f|^{2}-\frac{|f|^{2}}{2 \pi} \int_{0}^{2 \pi}\left(2 u \bar{f} f^{\prime}+\phi^{\prime} \overline{f f}\right) z d \theta
$$

and the condition $h^{\prime}(0)=0$ becomes, on applying Fubini's Theorem,

$$
\frac{1}{2 \pi} \int_{0}^{2 \pi} 2 u|f|^{2} e^{\alpha|f|^{2}} d \theta=\frac{S_{\alpha}(f)}{2 \pi} \int_{0}^{2 \pi}\left(2 u \bar{f} f^{\prime}+\phi^{\prime} f \bar{f}\right) z d \theta .
$$

That completes the proof.

COROLlaRy 1. Under the same assumptions on $f$ and $\phi$, we have

$$
\begin{aligned}
& \frac{1}{2 \pi} \int_{0}^{2 \pi}|f|^{2} e^{\alpha|f|^{2}}(\phi+\bar{\phi}) d \theta \\
& \quad=\frac{S_{\alpha}(f)}{2 \pi} \int_{0}^{2 \pi}\left\{(\phi+\bar{\phi}) f^{\prime} \bar{f} z+\phi\left(\overline{z f^{\prime}} f-z f^{\prime} \bar{f}\right)\right\} d \theta .
\end{aligned}
$$


Proof. This follows on integrating

$$
\int_{0}^{2 \pi} \phi^{\prime}\left(e^{i \theta}\right) f\left(e^{i \theta}\right) \overline{f\left(e^{i \theta}\right)} e^{i \theta} d \theta
$$

by parts, noting that $\frac{\partial}{\partial \theta} f\left(e^{i \theta}\right)=i e^{i \theta} f^{\prime}\left(e^{i \theta}\right)$, and that $\frac{\partial}{\partial \theta} \overline{f\left(e^{i \theta}\right)}=$ $-i e^{-i \theta} \overline{f^{\prime}\left(e^{i \theta}\right)}$.

CoRollary 2. Under the same assumptions on $f$ and $\phi$, we have

$$
\begin{aligned}
& \frac{1}{2 \pi} \int_{0}^{2 \pi}\left|f\left(e^{i \theta}\right)\right|^{2} e^{\alpha\left|f\left(e^{i \theta}\right)\right|^{2} \phi\left(e^{i \theta}\right) d \theta} \\
& \quad=\frac{S_{\alpha}(f)}{2 \pi} \int_{0}^{2 \pi} e^{-i \theta} \overline{f^{\prime}\left(e^{i \theta}\right)} f\left(e^{i \theta}\right) \phi\left(e^{i \theta}\right) d \theta .
\end{aligned}
$$

Proof. From Corollary 1, we have

$$
\begin{aligned}
& \frac{1}{2 \pi} \int_{0}^{2 \pi}|f|^{2} e^{\alpha|f|^{2}}(\phi+\bar{\phi}) d \theta \\
& \quad=\frac{S_{\alpha}(f)}{2 \pi} \int_{0}^{2 \pi}\left\{(\phi+\bar{\phi}) f^{\prime} \bar{f} z+\phi\left(\overline{z f^{\prime}} f-z f^{\prime} \bar{f}\right)\right\} d \theta .
\end{aligned}
$$

Replacing $\phi$ by $i \phi$ and then dividing by $i$ gives

$$
\begin{aligned}
& \frac{1}{2 \pi} \int_{0}^{2 \pi}|f|^{2} e^{\alpha|f|^{2}}(\phi-\bar{\phi}) d \theta \\
& \quad=\frac{S_{\alpha}(f)}{2 \pi} \int_{0}^{2 \pi}\left\{(\phi-\bar{\phi}) f^{\prime} \bar{f} z+\phi\left(\overline{z f^{\prime}} f-z f^{\prime} \bar{f}\right)\right\} d \theta .
\end{aligned}
$$

Adding these two equations completes the proof.

In the next corollary $\mathscr{H}_{0}^{p}$ denotes the set of boundary functions $f\left(e^{i \theta}\right)$ of functions $f \in H_{0}^{p}$, where $H_{0}^{p}$ denotes the set of functions in $H^{p}$ which vanish at the origin [cf. 6, §3.2].

COROLlary 3. If $f$ is as above, then

$$
|f|^{2} e^{\alpha|f|^{2}}-S_{\alpha}(f) \overline{f f^{\prime}} \in \mathscr{H}_{0}^{1} .
$$

It is easy to see that the identity function $f(z)=z$ satisfies the condition of Corollary 3. However, the argument above is not delicate enough to yield a uniqueness result. Indeed, if we consider the functionals

$$
J_{n}(f)=\frac{1}{2 \pi} \int_{0}^{2 \pi}\left|f\left(e^{i \theta}\right)\right|^{2 n} d \theta
$$


for $n=1,2, \ldots$, then essentially the same argument shows that an extremal function $f$ with $f^{\prime} \in H^{1}$ must satisfy

$$
|f|^{2 n}-J_{n}(f) \overline{f f^{\prime}} \in \mathscr{H}_{0}^{1}
$$

and again this condition is fulfilled by the identity function. Let

$$
\mu_{n}=\sup \left\{J_{n}(f) \mid\|f\|_{\mathfrak{D}} \leq 1\right\} \text {. }
$$

Because the $J_{n}$ are weakly continuous on the unit ball $B$ of $\mathfrak{D}$, extremal functions exist for each $n$. For $n=1$ and $f(z)=\sum_{n=1}^{\infty} a_{n} z^{n}$, we have

$$
J_{1}(f)=\sum_{n=1}^{\infty}\left|a_{n}\right|^{2} \leq \sum_{n=1}^{\infty} n\left|a_{n}\right|^{2}=\|f\|_{\mathfrak{D}}^{2},
$$

with equality if and only if $f(z)=a_{1} z$. Hence $\mu_{1}=1$ and the identity function is extremal. For $n=2$ a more delicate argument again shows that $\mu_{2}=1$ and the identity function is extremal. However, since there are unbounded functions in $\mathfrak{D}, \mu_{n} \rightarrow \infty$ as $n \rightarrow \infty$. But $J_{n}(f)=1$ for $f(z)=z$, so the identity function cannot be extremal for large $n$.

4. A local maximum. In this section we show that, in a certain sense, the identity function is a local maximum for each of the functionals $\Lambda_{\alpha}, 0<\alpha \leq 1$. For simplicity of exposition we restrict ourselves to functions with nonnegative coefficients. The idea is to analyze $\Lambda_{\alpha}$ along curves

$$
f_{t}(z)=z \cos t+h(z) \sin t, \quad 0 \leq t \leq \frac{\pi}{2},
$$

where $h \in \mathfrak{D},\|h\|_{\mathfrak{D}}=1$, and $h(z)=\sum_{n=2}^{\infty} b_{n} z^{n}$ has nonnegative coefficients. Since $z$ is orthogonal to $h(z),\left\|f_{t}\right\|_{\mathfrak{D}}=1$, and it is clear that any function in $\mathfrak{D}$ with norm one and nonnegative coefficients has such a representation.

For reasons which will become clear shortly, it will be convenient to treat the function $h(z)=\frac{1}{\sqrt{2}} z^{2}$ as a special case. In this case we have

$$
f_{t}(z)=z \cos t+\frac{1}{\sqrt{2}} z^{2} \sin t, \quad 0 \leq t \leq \frac{\pi}{2} .
$$

We let $\phi_{\alpha}(t)=e^{-\alpha} \Lambda_{\alpha}\left(f_{t}\right)$. 
Proposition 2. The function $\phi_{\alpha}(t)$ is strictly decreasing for $0 \leq t \leq$ $\frac{\pi}{2}, 0<\alpha \leq 1$.

Proof. Clearly,

$$
\left|f_{t}\left(e^{i \theta}\right)\right|^{2}=1-\frac{\sin ^{2} t}{2}+\sqrt{2} \sin t \cos t \cos \theta .
$$

Hence

$$
\phi_{\alpha}(t)=e^{-\alpha \frac{\sin ^{2} t}{2}} \frac{1}{2 \pi} \int_{0}^{2 \pi} e^{\sqrt{2} \alpha \sin t \cos t \cos \theta} d \theta
$$

But, for $n=0,1,2, \ldots$,

$$
\frac{1}{2 \pi} \int_{0}^{2 \pi} \cos ^{2 n+1} \theta d \theta=0
$$

and

$$
\frac{1}{2 \pi} \int_{0}^{2 \pi} \cos ^{2 n} \theta d \theta=\frac{(2 n) !}{2^{2 n}(n !)^{2}}
$$

Hence

$$
\begin{aligned}
& \frac{1}{2 \pi} \int_{0}^{2 \pi} e^{\sqrt{2} \alpha \sin t \cos t \cos \theta} d \theta \\
& \quad=\sum_{n=0}^{\infty} \frac{\alpha^{n}}{n !}(\sqrt{2} \sin t \cos t)^{n} \frac{1}{2 \pi} \int_{0}^{2 \pi} \cos ^{n} \theta d \theta \\
& \quad=\sum_{n=0}^{\infty} \frac{\alpha^{2 n}}{(2 n) !} 2^{n}\left(\sin ^{2} t \cos ^{2} t\right)^{n} \frac{(2 n) !}{2^{2 n}(n !)^{2}} \\
& =\sum_{n=0}^{\infty} \frac{\alpha^{2 n}}{(n !)^{2}}\left(\frac{\sin ^{2} t \cos ^{2} t}{2}\right)^{n} .
\end{aligned}
$$

Thus,

$$
\phi_{\alpha}(t)=e^{-\alpha \frac{\sin ^{2} t}{2}} \sum_{n=0}^{\infty} \frac{\alpha^{2 n}}{(n !)^{2}}\left(\frac{\sin ^{2} t \cos ^{2} t}{2}\right)^{n} .
$$

Differentiating $\phi_{\alpha}(t)$ with respect to $t$ gives 


$$
\begin{aligned}
\phi_{\alpha}^{\prime}(t)= & -\alpha \sin t \cos t e^{-\alpha \frac{\sin ^{2} t}{2}} \sum_{n=0}^{\infty} \frac{\alpha^{2 n}}{(n !)^{2}}\left(\frac{\sin ^{2} t \cos ^{2} t}{2}\right)^{n} \\
& +e^{-\alpha \frac{\sin ^{2} t}{2}} \sum_{n=0}^{\infty} \frac{n \alpha^{2 n}}{(n !)^{2}}\left(\frac{\sin ^{2} t \cos ^{2} t}{2}\right)^{n} \sin t \cos t\left(\cos ^{2} t-\sin ^{2} t\right) \\
= & \sin t \cos t e^{-\alpha \frac{\sin ^{2} t}{2}}\left[-\sum_{n=0}^{\infty} \frac{\alpha^{2 n+1}}{(n !)^{2}}\left(\frac{\sin ^{2} t \cos ^{2} t}{2}\right)^{n}\right. \\
& \left.+\left(\cos ^{2} t-\sin ^{2} t\right) \sum_{n=1}^{\infty} \frac{n \alpha^{2 n}}{(n !)^{2}}\left(\frac{\sin ^{2} t \cos ^{2} t}{2}\right)^{n-1}\right] \\
= & \sin t \cos t e^{-\alpha \frac{\sin ^{2} t}{2}}\left[-\sum_{n=0}^{\infty} \frac{\alpha^{2 n+1}}{(n !)^{2}}\left(\frac{\sin ^{2} t \cos ^{2} t}{2}\right)^{n}\right. \\
& \left.+\left(\cos ^{2} t-\sin ^{2} t\right) \sum_{n=0}^{\infty} \frac{\alpha^{2 n+2}}{(n !)^{2}(n+1)}\left(\frac{\sin ^{2} t \cos ^{2} t}{2}\right)^{n}\right] .
\end{aligned}
$$

But $\cos ^{2} t-\sin ^{2} t=\cos 2 t$, so

$$
\phi_{\alpha}^{\prime}(t)=-\sin t \cos t e^{-\alpha \frac{\sin ^{2} t}{2}} \sum_{n=0}^{\infty}\left(1-\frac{\alpha \cos 2 t}{n+1}\right) \frac{\alpha^{2 n+1}}{(n !)^{2}}\left(\frac{\sin ^{2} t \cos ^{2} t}{2}\right)^{n} .
$$

But $1-\frac{\alpha \cos 2 t}{n+1}>0$ unless $n=0, \alpha=1$, and $t=0$. That completes the proof.

Now if $h(z)=\sum_{n=2}^{\infty} b_{n} z^{n}$, we have

$$
\|h\|_{2}^{2}=\sum_{n=2}^{\infty} b_{n}^{2} \leq \frac{1}{2} \sum_{n=2}^{\infty} n b_{n}^{2}=\frac{1}{2}
$$

with equality if and only if $h(z)=\frac{1}{\sqrt{2}} z^{2}$. Having disposed of this case in Proposition 2, we may assume that $\|h\|_{2}^{2}<\frac{1}{2}$. Once again we let $\phi_{\alpha}(t)=e^{-\alpha} \Lambda_{\alpha}\left(f_{t}\right)$.

Proposition 3. For each $h$ there is a $T$ such that $\phi_{\alpha}(t)$ is decreasing for $0<t<T$.

Proof. If we write $k(z)=z h(\bar{z})+\bar{z} h(z)$, we obtain

$$
\left|f_{t}(z)\right|^{2}=1+\left(|h(z)|^{2}-1\right) \sin ^{2} t+k(z) \frac{\sin 2 t}{2} .
$$


On the other hand, applying Taylor's Theorem to the function

$$
q_{\alpha}(t)=e^{\alpha\left[(\lambda-1) \sin ^{2} t+\mu \frac{\sin 2 t}{2}\right]}
$$

gives

$$
q_{\alpha}(t)=1+\alpha \mu t+\alpha\left(\frac{\alpha \mu^{2}}{2}+\lambda-1\right) t^{2}+g_{\alpha}(\lambda, \mu, \tau) q_{\alpha}(\tau) t^{3},
$$

where $g_{\alpha}(\lambda, \mu, t)$ is a polynomial in $\alpha, \lambda$, and $\mu$, with coefficients depending only on $\sin 2 t$ and $\cos 2 t$, and $\tau$ is a number between 0 and $t$. Applying this to $e^{\alpha\left|f_{t}\right|^{2}}$ gives

$$
\begin{aligned}
e^{\alpha\left|f_{t}(z)\right|^{2}=e^{\alpha}(1+\alpha k(z) t+\alpha} & \left.\frac{k^{2}(z)}{2}+|h(z)|^{2}-1\right) t^{2} \\
& \left.+g_{\alpha}\left(|h(z)|^{2}, k(z), \tau\right) e^{\alpha\left|f_{\tau}(z)\right|^{2}} t^{3}\right) .
\end{aligned}
$$

Since $|k(z)| \leq 2|h(z)|$, and $\left|f_{\tau}\right|^{2} \leq 1+|h|^{2}$, the coefficient of $t^{3}$ is dominated by $p\left(|h|^{2}\right) e^{\alpha|h|^{2}}$, where $p$ is some polynomial. As remarked in the introduction, there is a constant $C_{h}$ such that

$$
\left|\frac{1}{2 \pi} \int_{0}^{2 \pi} p\left(\left|h\left(e^{i \theta}\right)\right|^{2}\right) e^{\alpha\left|h\left(e^{i \theta}\right)\right|^{2}} d \theta\right| \leq C_{h}
$$

Next, for $z=e^{i \theta}$, it is easy to see that

$$
k(z)=2 \sum_{n=1}^{\infty} b_{n+1} \cos n \theta
$$

so that

$$
\frac{1}{2 \pi} \int_{0}^{2 \pi} k\left(e^{i \theta}\right) d \theta=0
$$

and

$$
\frac{1}{2 \pi} \int_{0}^{2 \pi} k^{2}\left(e^{i \theta}\right) d \theta=2 \sum_{n=2}^{\infty} b_{n}^{2}=2\|h\|_{2}^{2} .
$$

Thus, integrating $e^{\alpha\left|f_{t}\left(e^{i \theta}\right)\right|^{2}}$ gives

$$
\phi_{\alpha}(t)=1+\alpha\left\{\|h\|_{2}^{2}(\alpha+1)-1\right\} t^{2}+c(t) t^{3},
$$


where $|c(t)| \leq C_{h}$. Since $\|h\|_{2}^{2}<\frac{1}{2}$ by assumption, it follows that $\phi_{\alpha}(t)$ is decreasing for $t$ close to 0 .

5. Concluding remarks. Beurling [1] proved that his estimate is sharp in the sense that for the functions

$$
B_{a}(z)=\left(\log \frac{1}{1-a z}\right) / \sqrt{\log \frac{1}{1-a^{2}}}, \quad 0<a<1,
$$

there is a constant $c$ independent of $a$ such that

$$
\left|E_{t}\right| \geq c e^{-t^{2}} \quad \text { if } t=\sqrt{\log \frac{1}{1-a^{2}}},
$$

and Chang and Marshall [5] proceed by comparing $f \in \mathfrak{D},\|f\|_{\mathfrak{D}}=1$, with an appropriate $B_{a}$. We note that

$$
B_{a}(z)=\sum_{n=1}^{\infty} \frac{a^{n} z^{n}}{n \sqrt{N_{a}}}
$$

where

$$
N_{a}=\log \frac{1}{1-a^{2}} .
$$

Since, as $a \rightarrow 0, \frac{1}{a} \sqrt{N_{a}} \rightarrow 1$, it follows that $B_{a}(z) \rightarrow z$ weakly as $a \rightarrow 0$. On the other hand, it can be shown that $B_{a}(z) \rightarrow 0$ weakly as $a \rightarrow 1$. It is of interest to investigate the behavior of $\Lambda_{1}\left(B_{a}\right)$. Numerical experiments suggest that $\Lambda_{1}\left(B_{a}\right)$ is decreasing and concave for $0<a<1$, and that $\lim _{a \rightarrow 0} \Lambda_{1}\left(B_{a}\right)=e$ and that $\lim _{a \rightarrow 1} \Lambda_{1}\left(B_{a}\right)=$ 0 . It is also possible to prove that $J_{1}\left(B_{a}\right)$ is decreasing and concave for $0<a<1$. However, numerical experiments suggest that this is not the case for $J_{n}\left(B_{a}\right)$ for larger $n$, and recently the second author has succeeded in proving that $\lim \sup _{a \rightarrow 1} \Lambda_{1}\left(B_{a}\right)>1$.

Finally, let

$$
S_{n, \alpha}(f)=\sum_{k=0}^{n} \frac{\alpha^{k} J_{k}(f)}{k !}
$$

denote the partial sums in the series expansion of $\Lambda_{\alpha}(f)$. We remark that, for fixed $\alpha$, if the identity function $f(z)=z$ is extremal for $S_{n, \alpha}$ for large $n$, then it would also be extremal for $\Lambda_{\alpha}$. Similarly, if $f(z)=z$ is extremal for $\Lambda_{\alpha}$ for $\alpha$ close to 1 , it would also be extremal for $\Lambda_{1}$. We conjecture that the identity function is extremal in all of these cases. 


\section{REFERENCES}

[1] A. Beurling, Etudes sur une problème de majoration, Thèse, Almqvist and Wiksell, Uppsala, 1933.

[2] L. Carleson, A representation formula for the Dirichlet integral, Math. Zeitschrift, 73 (1960), 190-196.

[3] L. Carleson and S.-Y. A. Chang, On the existence of an extremal function for an inequality of J. Moser, Bull. Sci. Math., 2e série, 110 (1985), 113-127.

[4] S.-Y. A. Chang, Extremal functions in a sharp form of Sobolev inequality, Proceedings of the Int. Congress of Math., Berkeley, 1986, pp. 715-723.

[5] S.-Y. A. Chang and D. E. Marshall, A sharp inequality concerning the Dirichlet integral, Amer. J. Math., 107 (1985), 1015-1033.

[6] Peter L. Duren, Theory of $H^{p}$ Spaces, Academic Press, New York, 1970.

[7] Matts Essén, Sharp estimates of uniform harmonic majorants in the plane, Ark. Mat., 25 (1987), 15-28.

[8] D. E. Marshall, A new proof of a sharp inequality concerning the Dirichlet integral, Arkiv för Mat., 27 (1989), 131-137.

[9] J. Moser, A sharp form of an inequality by $N$. Trudinger, Indiana Univ. Math. J., 20 (1971), 1077-1092.

Received February 20, 1992 and in revised form October 7, 1992.

LAMAR UNIVERSITY

BeAumont, TEXAs 77710

E-mail address: matheson@cs4.lamar.edu 


\title{
PACIFIC JOURNAL OF MATHEMATICS
}

\author{
Founded by
}

\author{
E. F. BECKENBACH (1906-1982) F. Wolf (1904-1989)
}

\section{EDITORS}

Sun-Yung A. Chang

(Managing Editor)

University of California

Los Angeles, CA 90024-1555

chang@math.ucla.edu

\section{F. Michael Christ}

University of California

Los Angeles, CA 90024-1555

christ@math.ucla.edu

Herbert Clemens

University of Utah

Salt Lake City, UT 84112

clemens@math.utah.edu
THOMAS ENRIGHT

University of California, San Diego

La Jolla, CA 92093

tenright@ucsd.edu

Nicholas ERCOLANI

University of Arizona

Tucson, AZ 85721

ercolani@math.arizona.edu

R. FINN

Stanford University

Stanford, CA 94305

finn@gauss.stanford.edu

VAUGHAN F. R. JONES

University of California

Berkeley, CA 94720

vfr@math.berkeley.edu
SteVen KerCKHofF

Stanford University

Stanford, CA 94305

spk@gauss.stanford.edu

MARTIN ScharLemanN

University of California

Santa Barbara, CA 93106

mgscharl@math.ucsb.edu

Harold STARK

University of California, San Diego

La Jolla, CA 92093

V. S. VARADARAJAN

University of California

Los Angeles, CA 90024-1555

vsv@math.ucla.edu

\section{SUPPORTING INSTITUTIONS}

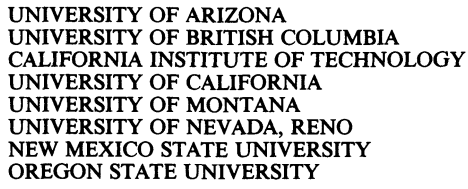

The Supporting Institutions listed above contribute to the cost of publication of this Journal, but they are not owners or publishers and have no responsibility for its content or policies.

Mathematical papers intended for publication in the Pacific Journal of Mathematics should be in typed form or offset-reproduced (not dittoed), double spaced with large margins. Please do not use built up fractions in the text of the manuscript. However, you may use them in the displayed equations. Underline Greek letters in red, German in green, and script in blue. The first paragraph must be capable of being used separately as a synopsis of the entire paper. In particular it should contain no bibliographic references. Please propose a heading for the odd numbered pages of less than 35 characters. Manuscripts, in triplicate, may be sent to any one of the editors. Please classify according to the 1991 Mathematics Subject Classification scheme which can be found in the December index volumes of Mathematical Reviews. Supply name and address of author to whom proofs should be sent. All other communications should be addressed to the managing editor, or Julie Honig, University of California, Los Angeles, California 90024-1555.

There are page-charges associated with articles appearing in the Pacific Journal of Mathematics. These charges are expected to be paid by the author's University, Government Agency or Company. If the author or authors do not have access to such Institutional support these charges are waived. Single authors will receive 75 free reprints; joint authors will receive a total of 100 free reprints. Additional copies may be obtained at cost in multiples of 50 .

The Pacific Journal of Mathematics (ISSN 0030-8730) is published monthly except for July and August. Regular subscription rate: $\$ 215.00$ a year (10 issues). Special rate: $\$ 107.50$ a year to individual members of supporting institutions.

Subscriptions, orders for numbers issued in the last three calendar years, and changes of address should be sent to Pacific Journal of Mathematics, P.O. Box 4163, Berkeley, CA 94704-0163, U.S.A. Old back numbers obtainable from Kraus Periodicals Co., Route 100, Millwood, NY 10546.

The Pacific Journal of Mathematics at University of California, c/o Department of Mathematics, 981 Evans Hall, Berkeley, CA 94720 (ISSN 0030-8730) is published monthly except for July and August. Second-class postage paid at Berkeley, CA 94704, and additional mailing offices. POSTMASTER: send address changes to Pacific Journal of Mathematics, P.O. Box 4163, Berkeley, CA 94704-0163.

PUBLISHED BY PACIFIC JOURNAL OF MATHEMATICS at University of California, Berkeley, CA 94720, A NON-PROFIT CORPORATION

This publication was typeset using $\mathcal{A} \mathcal{M} S-\mathrm{T}_{\mathrm{E}} \mathrm{X}$, the American Mathematical Society's $\mathrm{T}_{\mathrm{E}} \mathrm{X}$ macro system. Copyright (c) 1994 by Pacific Journal of Mathematics 


\section{PACIFIC JOURNAL OF MATHEMATICS}

Volume $162 \quad$ No. $2 \quad$ February 1994

On the existence of convex classical solutions to multilayer fluid

201 problems in arbitrary space dimensions

ANDREW FRENCH ACKER

Extremal functions and the Chang-Marshall inequality

VALENTIN V. ANDREEV and ALEC LANE MATHESON

Productive polynomials

RICHARD ARENS

On factor representations of discrete rational nilpotent groups and the 261 Plancherel formula

LAWRENCE JAY CORWIN and CAROLYN PFEFFER JOHNSTON

Commutants of Toeplitz operators on the Bergman space

ZELJKO CUCKOVIC

When $L^{1}$ of a vector measure is an AL-space

GUILlERMO P. CURBERA

A convexity theorem for semisimple symmetric spaces

305

KARL-HERMANN NEEB

Ideals of finite codimension in free algebras and the fc-localization

AMNON RosenmanN and ShMUEL Rosset

Dec groups for arbitrarily high exponents

BHARATH Al SETHURAMAN

Errata to: "The set of primes dividing the Lucas numbers has density $2 / 3$ "

JEFFREY C. LAGARIAS 